\title{
Structural basis of higher-order assembly formation in Toll-like receptors 1,2 and 6 signaling pathway
}

\author{
Yan Li \\ The University of Queensland, St Lucia, Australia; \\ yan.li9@uqconnect.edu.au
}

Innate immunity represents a typical and widely distributed form of immunity. Innate immune responses are the first line of defense against pathogens, which can help destroy invaders invertebrate animals, invertebrates, and plants. The innate immune system recognizes microorganisms via pattern-recognition receptors (PRRs). The family of Toll-like receptors (TLRs) is a distinct group of PRRs. They detect the microbial components known as pathogen-associated molecular patterns (PAMPs), activate downstream transcription factors such as nuclear factor- $\kappa \mathrm{B}(\mathrm{NF}-\kappa \mathrm{B})$, resulting in a pro-inflammatory response [1]. 10 TLRs has been identified in the human TLR family. In humans, TLR2 can form heterodimers with TLR1 and TLR6 when binding different types of ligands. The cytoplasmic Toll/interleukin-1 receptor (TIR) domain can be found in all TLRs and is responsible for transmitting extracellular signals to intracellular cytoplasmic TIR domain-containing adaptor proteins through TIR: TIR domain interactions, thus initiating downstream signaling. Two TIR-domain containing adaptor proteins, Myeloid differentiation primary response 88 (MyD88) and MyD88 adaptor-like (MAL) mediate downstream signaling in TLR2-TLR1/6 signaling pathway. It has been previously demonstrated that higher-order assembly formation occurs in the TLR4 signalling pathway [2]. The mechanism, which is known as signaling by cooperative assembly formation (SCAF), may occur in all TLR signal transduction. To date, the transduction mechanisms of TLR2TLR1/6 signalling are still unclear. My project is to determine the structural basis of higher-order assemblies formed by TIR domains with a focus on assemblies in the TLR2-TLR1/6 signalling.

Keywords: Toll-like receptors, Negative-stain EM, TLR signaling 